\title{
Peripheral Levels of EPCs, T Cells, and Macrophages' as Possible Predictive Biomarkers of Acute Myocardial Infarction
}

\section{Amankeldi Salybekov}

Shonan Kamakura General Hospital

\section{Katsuaki Sakai}

Tokai University Hospital

\section{Makoto Natsumeda}

Tokai University Hospital

\section{Kosit Vorateera}

Tokai University Hospital

\section{Shuzo Kobayashi}

Shonan Kamakura General Hospital

\section{Yuji Ikari}

Tokai University Hospital

Takayuki Asahara ( $\square$ asa777@is.icc.u-tokai.ac.jp )

Tokai University Hospital

\section{Research Article}

Keywords: acute myocardial infarction, biomarkers, endothelial progenitor cells, M1/M2 macrophages, T cells

Posted Date: November 24th, 2020

DOI: https://doi.org/10.21203/rs.3.rs-107073/v1

License: (c) (1) This work is licensed under a Creative Commons Attribution 4.0 International License. Read Full License 


\section{Peripheral Levels of EPCs, T Cells, and Macrophages' as}

3 Possible Predictive Biomarkers of Acute Myocardial

\section{Infarction}

\author{
Amankeldi A. Salybekov ${ }^{1,2}$, Katsuaki Sakai ${ }^{3}$, Makoto Natsumeda ${ }^{3}$, Kosit Vorateera ${ }^{1}$, Shuzo \\ Kobayashi ${ }^{2}$, Yuji Ikari ${ }^{3}$, and Takayuki Asahara ${ }^{1, *}$
}

1 Department of Advanced Medicine Science, Tokai University School of Medicine, 143 Shimokasuya, Isehara, Kanagawa 2591193, Japan.

2 Clinical Research Center, Shonan Kamakura General Hospital, 1370-1 Okamoto, Kamakura, Kanagawa, 2478533.

3 Department of Cardiology, Tokai University School of Medicine, 143 Shimokasuya, Isehara, Kanagawa, 2591193.

* Correspondence to T.A: asa777@is.icc.u-tokai.ac.jp

\section{Abstract}

Acute myocardial infarction (AMI), with a very relevant global disease burden, remains the major mortality and morbidity cause among all cardiovascular diseases. Patient prognosis is strictly dependent on early diagnosis and the adoption of adequate interventions. AMI diagnosis requires constant optimization, particularly considering the individuals at higher risk (or more vulnerable to worse outcomes) such as patients with diabetes mellitus and atherosclerosis. Herein, we investigated the levels of peripheral blood EPCs and immune cell-subsets from myeloid and lymphoid lineages, as well as their temporal dynamics, in the quest for new prognostic biomarkers of AMI. We collected blood from 18 hospitalized patients (days 3 and 7 after AMI onset) and 16 healthy volunteers, and resolved their circulating PBMC populations via flow cytometry. Overall, our data demonstrate a significant decrease in peripheral EPCs and CD8+ T cells, three days following an AMI. EPCs appear to be functionally impaired in AMI patients, and their circulating numbers associate with cardiac vessel lesions. Furthermore, CD8+ T cells (and even M1-macrophages) in the periphery, in combination with the classical laboratory determinations, may serve as high accuracy biomarkers of AMI, potentially aiding to prevent worse AMI outcomes. cells 
Acute myocardial infarction (AMI) is a pathological trigger that amplifies medullary hematopoiesis via sympathetic nervous system or damage-associated molecular pattern (DAMP) signaling ${ }^{1,2}$. Early mobilization and trafficking of stem cells to the site of injury seems to be important for an adequate cardiac repair response ${ }^{1,2}$. However, in patients with concomitant diseases/comorbidities or other important risk factors such as advanced age, the bone marrow stem cell niches become exhausted, potentially leading to worse clinical outcomes ${ }^{3,4,5}$. In fact, the prognostic value of circulating endothelial progenitor cells (EPCs) in patients with diabetes mellitus (DM) and atherosclerosis, among other diseases, is well documented. Continuously high blood glucose levels, elevated total cholesterol and their risk-associated factors such as obesity, smoking, and increasing age directly associate with the decrease in numbers and/or function of circulating EPCs, and correlate with increased mortality rates or the occurrence of major adverse cardiac events (MACE) ${ }^{6,7,8}$.

An increasing number of preclinical and clinical studies suggest that myeloid (M1- and M2-macrophages) and lymphoid (CD3, CD8, and CD4, among others) cells infiltrate into the ischemic tissue (including the myocardium) immediately after the onset of AMI and play an essential role in the resolution of acute inflammation, promoting cardiac healing ${ }^{9,10}$. Remarkably, temporal dynamic changes of human peripheral blood cells such as monocytes in the context of AMI have the potential to be used as early biomarkers for the prognosis of $\mathrm{AMI}^{11}$. Such a determination with prognostic value is even more important for patients with AMI in the context of problematic concomitant diseases such as DM and atherosclerosis or other cardiovascular disease (CVD) risk factors with associated worse outcomes. For instance, it would allow the correction of treatment schemes at the early phase of AMI, potentially preventing further complications.

In the present study, we investigated the levels of peripheral blood EPCs (CD34+) and immune cellsubsets from myeloid (M1-macrophages (CD11b+CD206-); and M2-macrophages (CD206+CD11b-)) and lymphoid (CD3+ pan $\mathrm{T}$ cell marker $(\mathrm{CD} 3+\mathrm{CD} 4+$; also known as $\mathrm{T}$-helper cells), cytotoxic $\mathrm{T}$ cells $(\mathrm{CD} 3+\mathrm{CD} 8+)$, and regulatory $\mathrm{T}$ cells $(\mathrm{CD} 3+\mathrm{CD} 4+\mathrm{CD} 127-\mathrm{CD} 25+))$ lineages, as well as their temporal dynamics, in the quest for new biomarkers of AMI (in combination with the clinical-laboratory findings).

\section{Materials and Methods}

\section{Subjects' Enrollment (Patients and Controls)}

Eighteen patients admitted to the Tokai University Hospital (Kanagawa, Japan), from September 2016 to December 2017, with the diagnosis of AMI were included in this study: 12 patients with ST-elevation myocardial infarction (STEMI), two patients with non-ST elevation myocardial infarction (NSTEMI) and two patients with unstable angina pectoris. All other patients with a history of chronic inflammatory disease and under anti-inflammatory medication were excluded from this study. Myocardial infarction was defined by the detection of "rise and fall" of cardiac troponin T (cTnT) and at least one of the following: symptoms of ischemia, new ST-T changes, or development of pathological Q-waves in the electrocardiogram. Immediately after hospitalization, patients underwent emergency coronary angiography and drug-eluting stents were deployed to their culprit lesions. The percutaneous coronary intervention (PCI), performed via the radial artery, was guided by optical coherence tomography (OCT) which allowed for the choice of stent size and length as well as proper stent positioning, and the pathological assessment of acute coronary syndromes. The OCT was performed prior to and post stent deployment. Non-culprit vessels were also screened using OCT to evaluate atherosclerotic 
pathophysiology. All patients received the standard AMI pharmacotherapy, including aspirin, P2Y 12 inhibitors, and low-molecular-weight heparin, according to usual hospital practice. Beta-blockers and angiotensin-converting enzyme inhibitors / angiotensin 2 receptor blockers were initiated post PCI, unless contraindicated, according to the AHA guidelines.

The control group consisted of sixteen healthy human volunteers aged under 60 years old, without overt cardiovascular diseases and DM, and were screened at least twice in the Tokai University Hospital outpatient clinic. Individuals with family history of dyslipidemia and AMI were not included in this study.

Clinical features were carefully recorded, including classical cardiovascular risk factors, age at diagnosis of DM (and duration, when applicable), body mass index (BMI), and glycosylated hemoglobin A1c (HbA1c), total-, LDL-, HDL-cholesterol, and triglycerides levels. The baseline characteristics of patients and healthy controls are shown in Sup. Table 1.

Specimen Collection and PBMCs Isolation

Peripheral blood (PB) (30-40 mL) was collected from hospitalized patients (days 3 and 7 after AMI onset) and healthy volunteers via venous puncture (in the forearm) using heparin-coated syringes. Peripheral mononuclear cells (PBMCs) were isolated by density gradient centrifugation using Histopaque $(d=1.077$; Sigma-Aldrich, St. Louis, MO, USA), as previously reported ${ }^{12,13}$. Briefly, immediately after centrifugation (400 g for 30 minutes at $20^{\circ} \mathrm{C}$ ), the mononuclear cell layer was carefully transferred to a new tube and further centrifuged ( $960 \mathrm{~g}$ for 20 minutes at $12{ }^{\circ} \mathrm{C}$ ). Then, the remaining red blood cells were lysed using $1 \mathrm{x}$ lysis buffer (BD, Franklin Lakes, NJ, USA), and the remaining platelets were removed by two centrifugation steps (200 g for 10 minutes at $12{ }^{\circ} \mathrm{C}$ ). Finally, PBMCs were resuspended and counted (cell viability per $1 \mathrm{~mL}$ of peripheral blood was recorded).

\section{Cell Phenotype Characterization}

Cell phenotype was evaluated by flow cytometry as reported elsewhere ${ }^{12,13}$, using the following antibodies (all from BioLegend, San Diego, CA, USA): anti-CD34 (clone 581), anti-CD133 (clone AC133), anti-CD11b (clone M1/70), anti-CD206 (clone 15-2), anti-CD11c (clone S-HCL-3), anti-CD3 (clone UCHT1), anti-CD4 (clone RPA-T4), anti-CD8 (clone SK1), anti-CD25 (clone BC96), and anti-CD127 (clone A019D5) (Supplementary table 1). PI was used in parallel as a viability marker. Briefly, cells were suspended in FACS buffer $\left(4 \times 10^{5}\right.$ cells $\left./ 200 \mu \mathrm{L}\right)$ and $1 \mu \mathrm{L}$ of FcR block (Miltenyi Biotec., Gladblach, Germany) was added and incubated at $4{ }^{\circ} \mathrm{C}$ for 20 minutes to reduce unspecific binding. Subsequently, the aforementioned antibodies were added, according to the manufacturer's manual, and left at $4{ }^{\circ} \mathrm{C}$ for 30 minutes. Stained cells were then washed twice and acquired using the BD FACS Verse or LSR Fortessa flow cytometers (BD, Franklin Lakes, NJ, USA). Raw data were analyzed using FlowJo (v. 10.6; FlowJo LLC, Ashland, OR, USA) and DeNovo (v. 6.2; DeNovo Ventures LLC, Boulder, CO, USA) software. For UMAP analysis, plugins were downloaded from FlowJo exchange and analyzed with R (version 3.6) and RStudio (version 1.2) tools. The PhenoGraph and

105 ClusterExplorer plugins were used to define distinct cell clusters by their true positivity level-comparison, immediately after myeloid and lymphoid cells subsets FCS files concatenation (after singlets and dead cells removal, based of FCS-H vs. FCS-A gating and PI signal, respectively). UMAP analysis was performed with 
and ClusterExplorer, then subsequently integrated with UMAP to reduce data dimension for an easier analysis of cell subsets.

Freshly isolated PBMCs $\left(1.5 \times 10^{5}\right)$ were seeded into semisolid methyl cellulose-based culture medium coated $35 \mathrm{~mm}$ dishes (Falcon, Corning, CA, USA) and left in a humidified incubator with $5 \% \mathrm{CO}_{2}$ at $37{ }^{\circ} \mathrm{C}$ until colony appearance. The number of adherent colonies was counted between days 16-18, using a gridded scoring dish (STEMCELL Tech., Vancouver, Canada) under a phase-contrast light microscope (Eclipse TE3000; Nikon, Tokyo, Japan). Primitive EPC colony-forming units (PEPC-CFUs) and definitive EPC colonyforming units (DEPC-CFUs) were separately counted as reported elsewhere ${ }^{12,13}$.

\section{Statistical Analysis}

All values are shown as mean \pm standard error. Statistical differences were investigated using the KruskalWallis with Benjamini, Krieger and Yekutieli post hoc analysis (to control for the false discovery rate). To correctly classify CD34+ cells count as a risk factor at different time points of AMI, we employed receiver operating characteristic (ROC) curve analysis. The area under the ROC curve (AUC) indicates the probability of correct classification or prediction: an AUC equal to 1 suggests perfect or strong classification, while an $\mathrm{AUC} \leq 0.5$ indicates random selection ${ }^{15}$. Correlations were evaluated using the Spearman rank correlation test. All statistical analyses were performed using the GraphPad Prism Software (v. 7c and 8.4; GraphPad, San Diego, CA, USA). Statistical significance was given by $\mathrm{P}<0.05$.

\section{Results}

\section{Reduction of EPCs is a Potential Early Biomarker of Cardiac Vessel-Lesions}

Flow cytometry analysis revealed different patterns with respect to the peripheral blood cells of AMI patients, at days 3 (Fig. 1A-B) post-AMI onset, compared to those of healthy individuals (Fig. 1C). Remarkably, the cell frequency of peripheral mononuclear live cells was significantly lower in patients on days 3 and 7 postAMI onset compared with healthy controls (Fig. 1D; $55.18 \pm 6.7$ vs. $77.5 \pm 2, \mathrm{P}<0.01$; and $64.52 \pm 7.33$ vs. $77.5 \pm 2, \mathrm{P}>0.08$, respectively). As shown in Fig. 1E, the lowest frequency of total CD34+ cells were seen in the group of AMI patients, three days after AMI onset (AMI-D3). Furthermore, and as expected, at day seven after AMI (AMI-D7) CD34+ frequency peaked to values similar or slightly higher (without statistical significance) than those of the control group (Fig. 1E). To address which subsets of CD34+ cells were increased at day seven post-AMI onset, we evaluated the expression of the tissue-committed stem cell surface molecules $\mathrm{CD} 11 \mathrm{~b}+$ and $\mathrm{CD} 11 \mathrm{c}+$ within CD34+ cells. Interestingly, the CD34+CD11b-CD11c- EPC subset was significantly increased in AMI-D7 in comparison with the AMI-D3 group (Fig. 1F; $0.029 \pm 0.02$ vs.

$1421.535 \pm 1.15, \mathrm{P}<0.05$ ), indicating a delayed mobilization response of immature CD34+ cells in AMI. The correlation matrix of multiple immune cells followed by the Spearman's rank test demonstrated that at day three post-AMI onset, $\mathrm{CD} 34+$ cell numbers positively and inversely correlated with $\mathrm{CD} 8+\mathrm{T}(\mathrm{r}=0.75 ; \mathrm{P}<0.01)$ and 
146 cells are probably the first to respond (and mobilize to the injured tissue) following AMI; thus, leading to their

147 reduction in the peripheral blood cell pool. On the other hand, at day 7 post-AMI, no relevant correlation was

148 detected between EPCs and other cell subsets (Fig. 1H). A receiver-operating characteristic (ROC) curve

149 associated with logistic regression analysis demonstrated an area under the ROC curve (AUC) of 0.95 (Figure

$1501 \mathrm{I} ; \mathrm{P}<0.0007$ ) or $89 \%$ of sensitivity, for the distinction of CD34+ cell frequencies for AMI-D3 vs. healthy

151 controls, indicating some biomarker potential. On the other hand, when this comparison was done with the

152 AMI-D7 group, the predictive value of EPCs was lost: $\mathrm{AUC}=0.71$ with $66 \%$ sensitivity (Fig. 1J; $\mathrm{P}>0.16$ ). As

153 the AMI-D3 group showed a high-accuracy classifier rate, we investigated whether EPC numbers could be used

154 as early cardiac arterial damage biomarker following AMI. As a result, correlation analysis showed inversely

155 correlated coronary lesion numbers to total EPC numbers in the AMI-D3 group (Fig. 1K; $r=-0.45, P>0.09$ ),

156 but not in the AMI-D7 group ( $\mathrm{r}=0.39, \mathrm{P}>0.33$ ) (Fig. S1A). Furthermore, we investigated whether there was a

157 linear trend between absolute EPCs and cardiac vessels lesion numbers at different time-points. The chi-square

158 test revealed a higher tendency for linear relation at day three vs. day seven post-AMI (Fig. S1B). In summary,

159 these results suggest that total EPC cell numbers $/ \mathrm{mL}$ of blood at day three post-AMI onset are potential

160 independent indicators/biomarker of cardiac vessel lesion. 


\section{Figure 1}
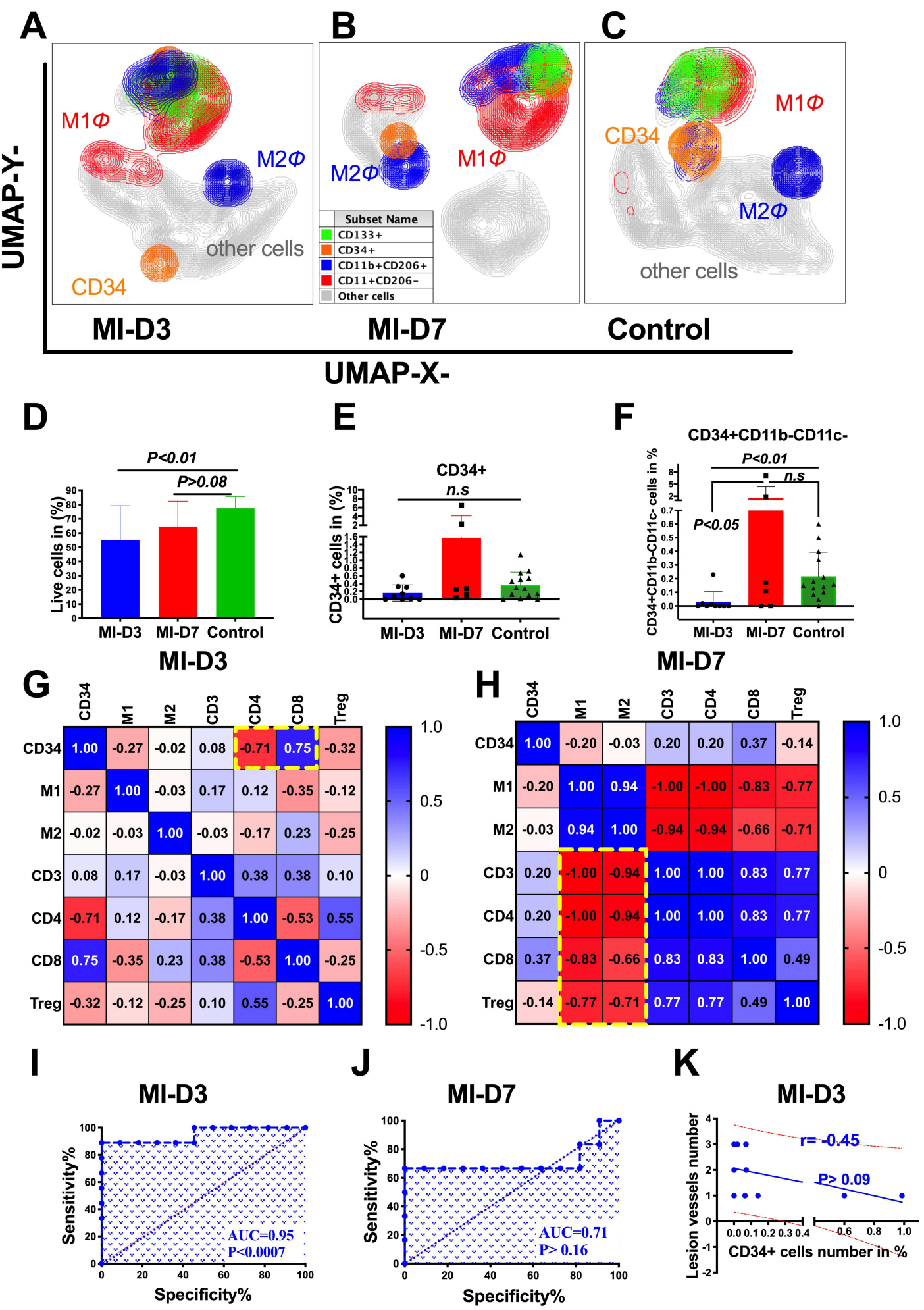

EPCs as a predictor of heart-vessel lesions. Unsupervised UMAP clustering method of immune cell populations were applied to distinguish myeloid and stem progenitor cells subsets at days three (A), and seven (B) after AMI onset, and in healthy controls (C). Peripheral blood live mononuclear cell frequencies were determined and are represented for the three groups (D). CD34+ cell frequencies are also represented (E), as are the ones for CD34+CD11b-CD11c- cells (F) for the three groups. Multivariable immune cells' correlation 
matrix analysis followed by the Spearman's rank test was performed and is represented for patient samples at days three $(\mathbf{G})$ and seven $(\mathbf{H})$ post-AMI onset. ROC curves were designed using CD34+ cells $/ \mathrm{mL}$ counts from AMI-D3 (I) and AMI-D7 patients (J) versus healthy controls. A correlation between CD34+ cell frequencies and cardiac vessel lesions in AMI-D3 patients was investigated and is represented (K). Data are represented as group mean \pm SEM. Statistical analysis was performed using the Kruskal-Wallis test followed by a Benjamini, Krieger and Yekutieli post hoc analysis. Abbreviations: AMI-D3, 3 days post-AMI onset; AMI-D7, 7 days postAMI onset; AMI, acute myocardial infarction; AUC, area under the receiver operating characteristics' curve

\section{M2 Macrophages Inversely Correlate with Troponin T, a Cardiac Necrosis Marker}

In line with our previous observation, the pie chart distribution of total PBMCs showed an increase in EPCs at day 7 post-AMI onset. (Fig. 2A; 5.15-fold vs. AMI-D3; 3-fold vs Control). In parallel, M1 or classical inflammatory monocyte/macrophages also dramatically increased at day seven post-AMI (Fig. 2A and B; 1.72fold vs MI-D3, $\mathrm{P}<0.004 ; 1.72$-fold vs Control, $\mathrm{P}<0.01$ ). On the other hand, alternatively activated or M2macrophages were slightly elevated in the AMI-D3 group but did not reach statistical significance (Fig. 2C).

180 This perhaps indicates that long-term elevated blood glucose and cholesterol levels may cause chronic

181 inflammation, and that the subsequent M2-macrophage increased as a regulatory response, as reported earlier ${ }^{16,18}$. Interestingly, using the Spearman correlation rank test, we observed that in AMI-D3 patients, M2macrophage numbers $/ \mathrm{mL}$ of blood (but not M1-macrophage) inversely correlated with the myocardial necrosis marker troponin $\mathrm{T}$ levels (Fig 2D; $\mathrm{r}=-0.73, \mathrm{P}<0.02$ ). However, this correlation was lost 7 days post-AMI (Fig 2E). We finally performed a ROC curve analysis, to evaluate the predictive potential of M1-macrophages in the context of AMI. As a result, while no significant findings were observed for AMI-D3 patients (Fig 2F; $\mathrm{AUC}=0.5, \mathrm{P}>0.8$ ), in AMI-D7 patients, M1-macrophages were defined as a potential biomarker (Fig 2G; $\mathrm{AUC}=0.86, \mathrm{P}<0.03)$.

To sum up, in the early phase of AMI, M2-macrophages increased and inversely correlated with cardiac troponin T, whereas peripheral M1-macrophages significantly increased at day seven, in response to AMI and combined with classical myocardial damage biomarkers may serve as a post-AMI biomarker. 
Figure 2
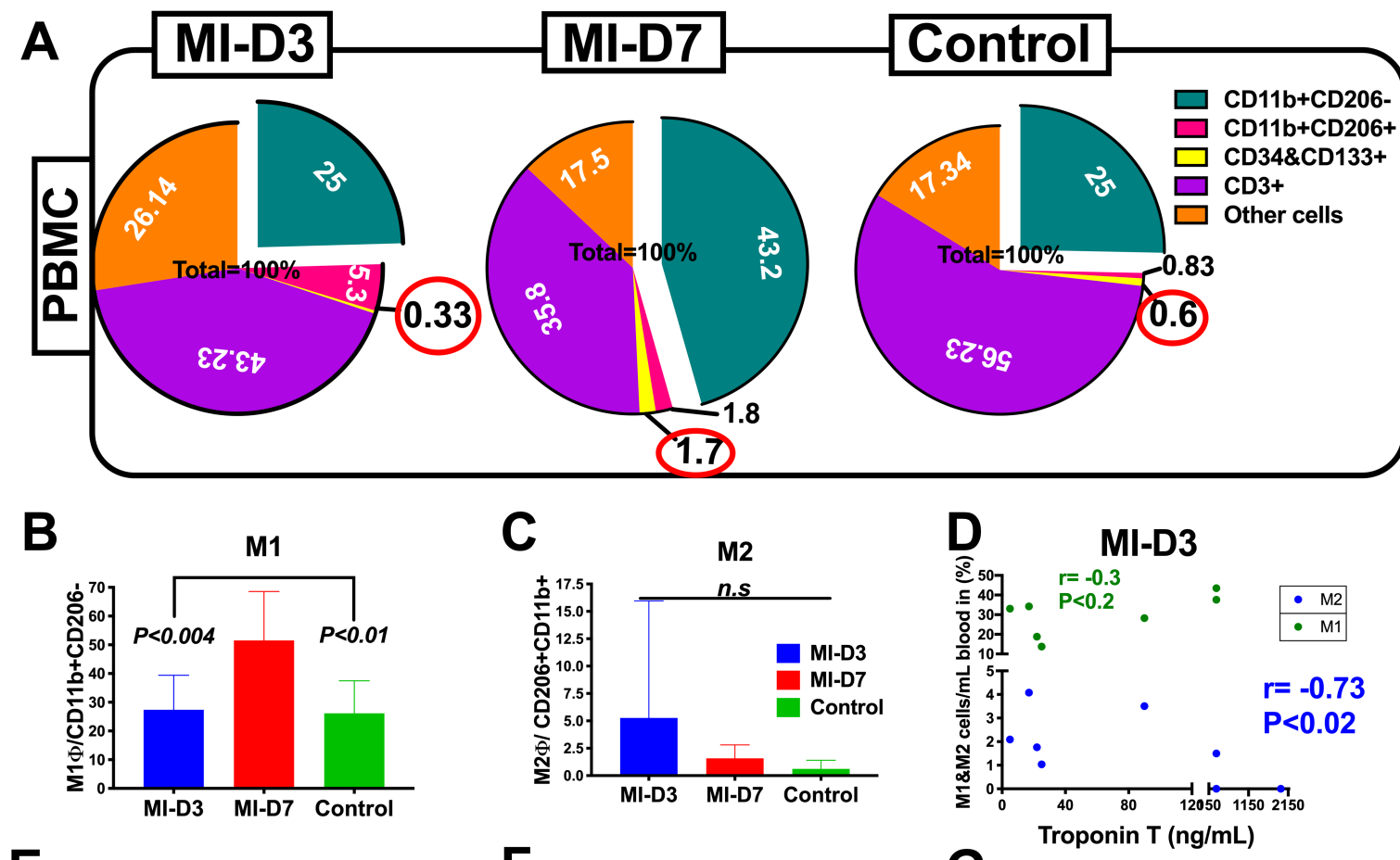

E

F

G
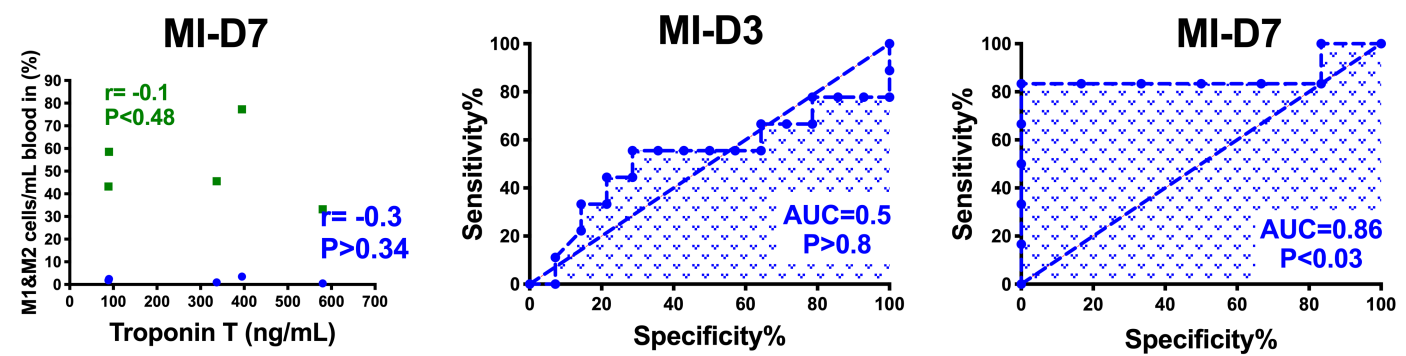

Macrophage subsets temporal dynamics in peripheral blood following AMI. Pie charts show myeloid cell subsets relative distribution over time in AMI patients, compared with healthy controls (A). Frequencies of (M2) alternatively activated (B) or (M1-) classical inflammatory (C) macrophages were determined and are represented. Macrophage subsets (M1 in green and M2 in blue) were correlated with the cardiac necrosis marker Troponin T levels at days three (D) and seven (E) after AMI onset. ROC curve analysis comparing M1macrophages from healthy individuals with AMI patients at days 3 (F) and 7 (G) post-AMI onset were performed and are represented. Data are shown as group means \pm SEM. Statistical analysis was performed using the Kruskal-Wallis test with Benjamini, Krieger and Yekutieli post hoc analysis. The Spearman rank correlation test was also used.

CD8+ T Cells in Circulation Decrease Early After AMI

As shown in the illustration graph obtained using UMAP, T cells, and the relative distribution their subsets undoubtedly declined in AMI patients compared with controls (Fig. 3A-C). Additionally, this distribution also slightly changed over time in AMI patients (Fig. 3A, B). Remarkably, total T cell frequency was significantly lower in AMI-D3 and AMI-D7 patients compared to healthy controls (Fig. 3D; $43.23 \pm 2.3$ vs. $56.23 \pm 2.6$, $\mathrm{P}<0.01$; and $35.8 \pm 6.18$ vs. $56.23 \pm 2.6, \mathrm{P}<0.01$, respectively). Interestingly, while no differences were 
209 (CD3+CD8-CD4+CD127-CD25+) frequencies (Fig. 3E and 3F, respectively), a significant drop was observed 210 in the frequency of cytotoxic T cells (CD3+CD4-CD8+) in AMI patients 3 and 7 days post-AMI, compared 211 with healthy controls (Fig. 3G; $9.1 \pm 1.8$ vs. $22.4 \pm 1.9, \mathrm{P}<0.002$; and $10.4 \pm 22.4, \mathrm{P}<0.01$, respectively). 212 Furthermore, when a correlation rank test was applied to understand the relation between $\mathrm{T}$ cells and the cardiac 213 necrosis marker creatine kinase myocardial band (CK-MB), a tendential positive correlation was observed for $214 \mathrm{CD} 8+\mathrm{T}$ cells, three days after AMI onset (Fig. 3H; r =0.58; $\mathrm{P}<0.054)$. However, this correlation was lost at 215 day seven post-AMI (Fig. 3I). Additionally, no relevant association was found for CD4+ $\mathrm{T}$ cells at both three216 and seven-days post-AMI (Fig. 3H, I). Next, we addressed if CD8+ T cells could be used as an early AMI 217 predictor, considering their relevant association with the cardiac necrosis marker at day three post-AMI. 218 Importantly, ROC curve analysis suggested that circulating CD8+ T cells would have a high accuracy of AMI 219 prediction, at days three (Fig. 3J, AUC $=0.94, \mathrm{P}<0.0004$ ) and seven (Fig. 3K, AUC $=0.85, \mathrm{P}<0.03$ ) post220 AMI. Notably, all $\mathrm{T}$ cell subsets strongly correlated (in a negative fashion) with myeloid cell subsets at day 221 seven post-AMI (Fig. 1H, yellow dashed box), suggesting that the T cell loss in the PB was compensated by 222 the increase of monocyte/macrophage cell subsets. Taken together, these results suggest that circulating CD8+ 223 T cells may serve as an early predictor of AMI. 


\section{Figure 3}
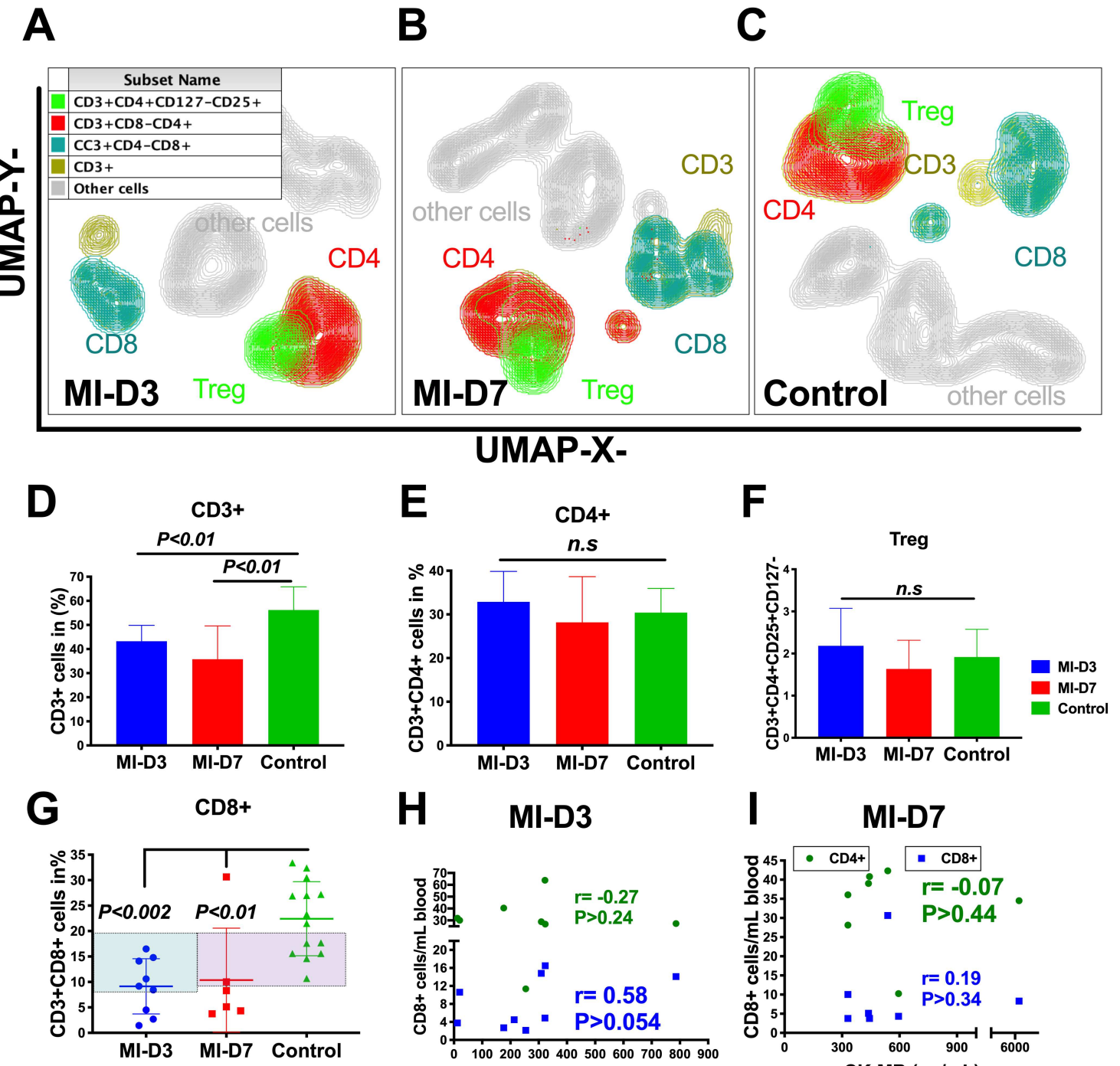

J

CK-MB (ng/mL)
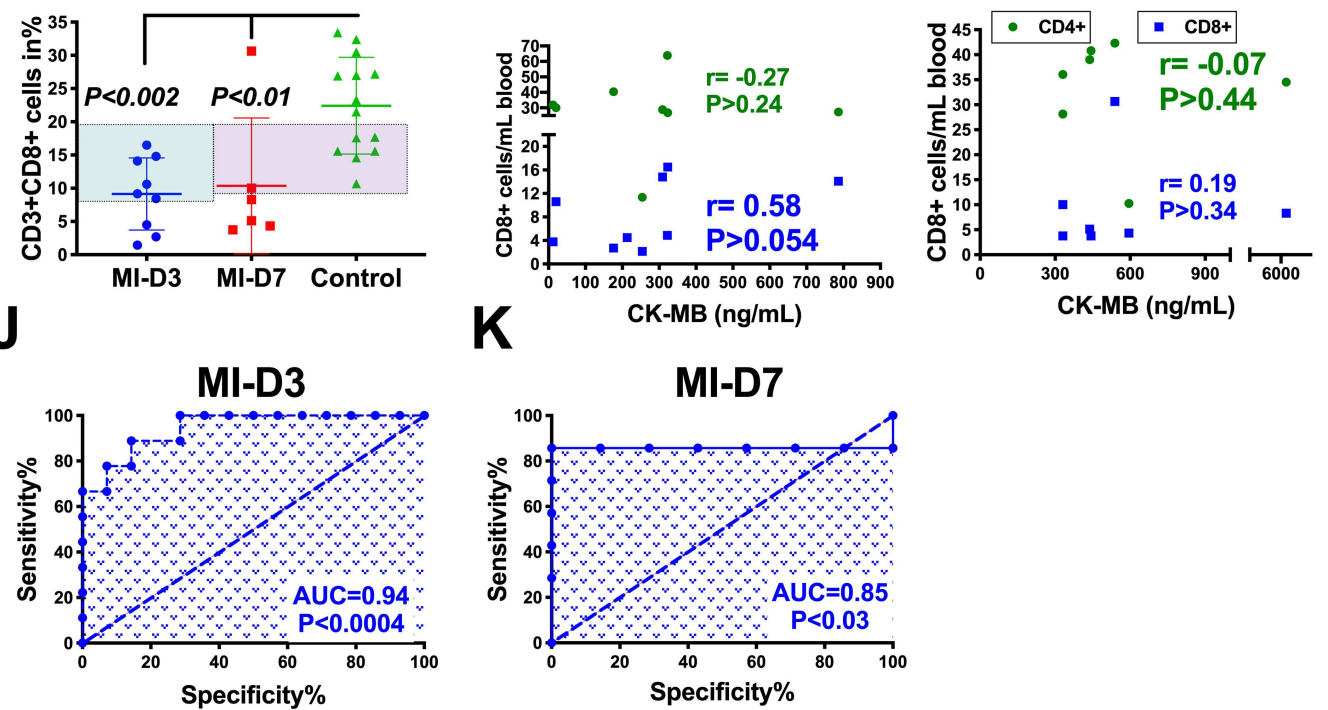

K

\section{MI-D7}

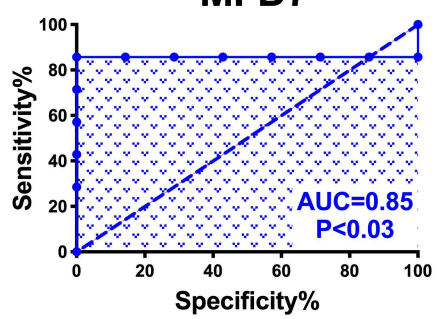

CD8 cells as early cardiac damage markers in AMI. The unsupervised UMAP clustering method of cell populations were applied to evaluate T cell subsets at days three (A), and seven (B) post-AMI, and in healthy controls $(\mathbf{C})$. Total $\mathrm{T}$ cell frequencies were determined in the three groups and are represented (D). Helper $\mathrm{T}$ cells, $(\mathbf{E})$ regulatory $\mathrm{T}$ cells $(\mathbf{F})$, and cytotoxic T cells' $(\mathbf{G})$ frequencies are also represented, per group. Correlation between CD4+ or CD8+ T and the cardiac necrosis marker CK-MB were investigated and are represented for the time-points of $3(\mathbf{H})$ and 7 (I) days after AMI onset. h). ROC curve analysis for CD8+ T cells is also represented for $3(\mathbf{J})$ and $7(\mathbf{K})$ days post-AMI onset. Data are shown as group means \pm SEM. Statistical analysis was performed using the Kruskal-Wallis test with Benjamini, Krieger and Yekutieli post hoc analysis. The spearman rank correlation test was also used. 
The endothelial progenitor cells in vitro colony-forming assay (EPC-CFA) revealed that colony-forming ability of cells from AMI patients collected 3- and 7-days post-AMI onset was tendentially lower compared with that from healthy controls (Fig. 4A-B). This was true considering either primitive EPC-CFU or definitive EPC-CFU (Fig. 4A-B). Particularly, the AMI-D3 group had the lowest DEPC-CFU (however non-significant compared to the control condition; $\mathrm{P}<0.05$ ), indicating a potentially lower vasculogenic EPC number, in line with the data obtained for CD34+ cells (Fig. 1E-F and Fig. 4B). This result may also suggest that EPCs were functionally impaired in our patients with risk factors. Furthermore, there was an increasing trend of EPC colony formation at day 7 post-AMI onset (compared with day 3), but without statistical relevance (Fig. 4B).

\section{Figure 4}

A

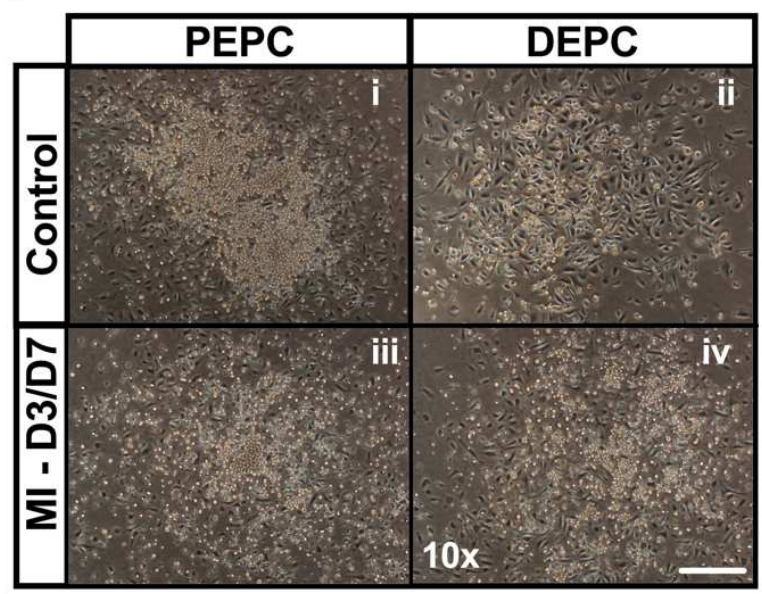

B

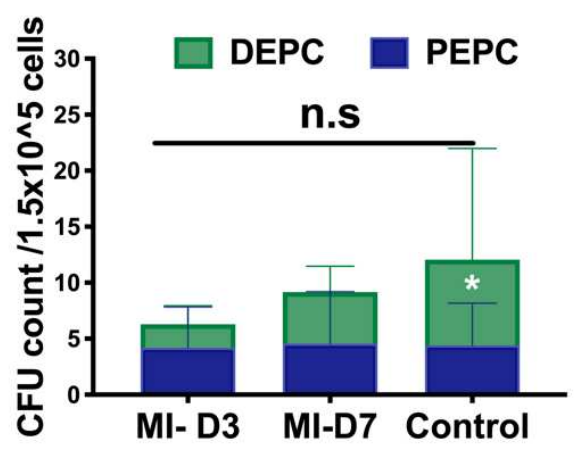

EPCs of an AMI patient show reduced colony formation ability. Colony-forming units of primitive EPC colonies (PEPC) and definitive EPC colonies (DEPC) were evaluated. PEPC and DEPC illustrative images are represented (A). Quantification and comparison of PEPC and DEPC among MI-D3, MI-D7, and Control groups is also represented $(\mathbf{B})$. Data are shown as group means \pm SEM. Statistical analysis was performed using the Kruskal-Wallis test with Benjamini, Krieger and Yekutieli post hoc analysis.

\section{Discussion}

In the present study, we evaluated EPCs, myeloid (M1 and M2-macrophages) and lymphoid (T cells and their subsets) cells in peripheral blood and their temporal dynamics in AMI patients (acute and subacute phases). Our principal findings are: 1) EPCs classify as strong coronary-lesion predictors, early after AMI together with clinical and laboratory findings such as glycated albumin and OCT; 2) T cell vs. myeloid cell ratios dynamically changed and indicated a decrease in circulating $\mathrm{T}$ cell with time and are compensated by myeloid cell subsets at day seven post-AMI onset; 3) CD8+ T cells may have high biomarker potential in the context of AMI with the combination of classical clinical and laboratory findings.

In our study, we observed a marked reduction in the viability of total PBMCs in AMI patients, both at days three and seven after AMI onset, compared to healthy controls. Previous studies have revealed that AMI patients show altered intracellular glutathione levels and an imbalance in the glutathione-system key enzymes glutamyl transferase and glutathione-S-transferase ${ }^{16}$. This, in conjunction with glycated end-products and "bad" 
cholesterol accumulation, may aggravate mitochondrial DNA damage and lead to telomere shortening, 262 promoting PBMC senescence ${ }^{16,17,19}$; our phenotype aligns with this idea. Furthermore, our correlation data 263 suggests that absolute EPC numbers inversely correlated with glycated albumin and MAGE, suggesting that 264 high glucose levels (and concomitant diseases or risk factors) may reduce EPCs in AMI patients (Fig. S1C).

265 Even the elevated frequency of immature EPCs we observed in AMI patients seven days after AMI onset was unable to generate EPC colonies to the same extent as healthy individuals. This may suggest that EPCs from AMI patients were functionally impaired. In our study, we focused on both primitive and definitive colonies. While PEPC are defined as very immature and highly proliferative colonies, DEPC are believed to derive from PEPC and represent cells prone to differentiation and promotion of vasculogenesis. Curiously, with respect to EPCs from our AMI-patients, along with the lower potential of colony formation, we observed a prevalence of PEPC (versus DEPC) early after AMI, which may suggest that neo-vascularization events may be compromised in AMI patients with. Aligning with this idea, recently, we showed that diet-induced obesity in mice led to the decrease of EPC kinetics in PB via EPC differentiation impairment. Interestingly, in the same model, pathologic ischemia stimulated EPC expansion in the bone marrow (BM). However, these cells were unable to egress to the bloodstream ${ }^{13}$, perhaps paralleling with the delayed EPCs mobilization we describe in the current study. Furthermore, at day seven post-AMI, the counts of PEPC and DEPC became indistinguishable, indicating that EPC proliferation was previously increased, preventing maturation to the functional endothelial cells, as we reported elsewhere ${ }^{13,20}$. Interestingly, Shintani et $\mathrm{al}^{21}$ also observed that at day seven post-pathologic ischemia stimuli in patients with AMI, EPCs reached their peak in peripheral blood (aligning with our results), suggesting a delayed EPCs mobilization response. In our multivariable correlation matrix analysis, CD34 cells positively correlated with CD8+ T cells (and inversely correlated with $\mathrm{T}$ helper cells). This may suggest that both CD34+ and CD8+ cells are highly sensitive to AMI events, making them distinguishable (among the all cells analyzed), and consequently potentially good biomarkers. Our ROC curve analysis clearly demonstrated that EPCs cell numbers $/ \mathrm{mL}$ are independent, highly accurate, and predictive indicators of cardiac vessel-lesions in the early phase of AMI (day three but not day seven). This observation was validated by the (inverse) correlation of cardiac vessel-lesion numbers (which was documented after PCI +OCT performance) and EPCs. Overall, herein, we clearly demonstrate the ability of EPCs to be used as a biomarker of heart vessel-lesions in the early phase of myocardial infarction.

Accumulating evidence demonstrates a role for hypercholesterolemia and hyperglycemia as factors which can independently regulate hematopoietic stem and progenitor cells (HSPC) function, or alter the phenotype of cells within the bone marrow niche; thus, indirectly influencing HSPC proliferation, maturation, and mobilization to the PB stream ${ }^{22}$. Additionally, there is an increasing interest in the role of chronic inflammatory stress such as DM and atherosclerosis, as modulators of $\mathrm{PBMCs}^{23,24}$. Our results showed that three days postAMI onset, M2-macrophage frequency was slightly higher, compared to AMI-D7 or control groups, which may imply that in CVD patients, M2-macrophages are elevated in the steady-state, as reported by others ${ }^{18}$. Once a pathological trigger occurs (such as AMI), these cells probably mobilize to the myocardial necrotic tissues to scavenge, as is suggested by the negative correlation we observed between M2-macrophages and troponin T, early after AMI (3 days' time-point). Notably, seven days after AMI onset, myeloid cells (M1 and M2299 macrophages) accounted for almost half of the total PBMCs (more than all the $\mathrm{T}$ cell subsets together). 300 Interestingly, this was mainly due to a significant increase in circulating M1-macrophages, seven days after 301 AMI onset. These observations may align with the notion that T cells (especially CD8+ cells) promote/need 
macrophage infiltration into the myocardium and their subsequent activation to express pro-inflammatory

303 cytokines and chemokines promoting acute cardiac inflammation ${ }^{25}$. In a previous study in vivo, using a murine

304 AMI model, we have shown that infiltration of M1-macrophages to the myocardium starts from day one and 305 peaks at day five after AMI onset, while M2-macrophages gradually increase from day three and peak at day 306 seven post-AMI onset ${ }^{9}$. However, the same was not observed when we induced AMI in obese mice (elevated 307 glucose and cholesterol levels): macrophages (M1 and M2) and T cells (CD4+, CD8+ and Tregs) dramatically 308 decreased by 3-fold in peripheral blood compared to the non-obese group immediately after the onset of 309 myocardial infarction (unpublished data). This drop in macrophage and T cell subsets continued up to day three 310 after AMI onset, gradually increasing afterward (unpublished data). Our ROC curve analysis showed that at 311 day seven (but not day three) post-AMI M1-macrophages biomarker potential (in the context of AMI) was 312 significant, while for $\mathrm{CD} 8 \mathrm{~T}$ cells this was true at both three $(\mathrm{AUC}=0.94)$ and seven $(\mathrm{AUC}=0.86)$ days post313 AMI onset. Several clinical and preclinical studies have shown that $\mathrm{T}$ cell recruitment to the ischemic 314 myocardium starts immediately following the onset of AMI. In fact, this was clearly demonstrated in a porcine 315 AMI mode ${ }^{26}$. Remarkably, this is particularly true for CD8+ T cells in a murine AMI model, where their early 316 emergence to the ischemic myocardium has been previously demonstrated ${ }^{9,26}$. Recently, Forteza et al. 317 demonstrated that in STEMI patients, T cells experienced a sharp decrease within 24 hours post-PCI, indicting 318 the early response of $\mathrm{T}$ cells to the aseptic necrotic myocardium ${ }^{26}$. Herein, we have appropriately dissected $\mathrm{T}$ 319 cells for their down subsets, and subsequently as outcomes revealed CD8 cells to potentially be reliable 320 biomarkers of AMI. The combination of CD8 cell absolute numbers and myocardium necrosis markers is 321 important and beneficial to differentiate atypical pneumonia or even upper abdominal acute inflammatory 322 diseases, which may exhibit similar clinical features as AMI. To date, the classical myocardial necrosis markers 323 of AMI such as troponin T or CK-MB are used as early diagnostic markers, peaking in PB 18-24 hour and 1232448 hour after AMI onset, respectively ${ }^{27}$. Early response of CD8+ T cells to ischemic injury increases its 325 utilization as an early diagnosis biomarker of AMI due to the maximum peaking of classical myocardium 326 necrosis biomarker level, one day after an AMI. Still, the huge burden of AMI (the main cause of mortality and 327 morbidity among all cardiovascular diseases), requires the constant optimization of AMI diagnosis and 328 treatment ${ }^{28}$. Our results strongly support the use of $\mathrm{CD} 8+\mathrm{T}$ cell counts as an early diagnostic biomarker of 329 AMI, in parallel with the classical cardiac necrosis markers, to improve diagnostic accuracy and sensitivity; this 330 determination may also have prognostic value. In a retrospective cohort study, lymphopenia among STEMI 331 patients was associated with a lower 3-year survival rate. Notably, CD8+ T cells decreased significantly and 332 strongly correlated with the fractalkine receptor CX3CR1 (contrary to CD4+ T cells) ${ }^{29}$. Other studies also 333 revealed the correlation of newly characterized $\mathrm{CD} 8+\mathrm{CD} 57+$ and $\mathrm{CD} 8+\mathrm{CD} 56+\mathrm{T}$ cell populations with 334 mortality, 6 months after AMI, and suggested the contribution of these specific CD8+ T cell subsets to acute 335 coronary events via their pro-inflammatory and high cytotoxic capacities ${ }^{30,31}$. In our study, other cell subsets 336 also increased in patients PB, 3 days after AMI onset (28\% compared to controls). On the other hand, compared 337 to healthy individuals, T cell populations decreased more than $12 \%$ and $16 \%$, three and seven days after AMI 338 onset, respectively (aligning with some abovementioned studies). Taken together, our results — consistent with 339 classical laboratory findings - indicate that mainly CD8+ T cells, but also M1-macrophage cell counts in PB 340 can be used as high accuracy biomarkers of AMI.

341 A few limitations of our study need to be acknowledged. First, our sample-size is relatively small, which 342 might have conditioned our statistical relevance, due to the intrinsic variations detected by flow cytometry 
analysis (method we used to quantify EPCs, macrophages, and T cell subsets). Second, patients who received statins and anti-diabetic treatments prior to an AMI were not analyzed separately (precisely because of our small sample-size), which may result in some type II errors: previous studies documented that DPP4-inhibitors and statins may affect hematopoiesis, as well as EPC number, kinetics, and mobilization ${ }^{32,33,34,35}$. Indeed, a large sample-size study is required to answer several critical points such as severe vs. mild MI, diabetes vs. nondiabetes, and gender differences. Nevertheless, our study attempts to show the significance of circulating blood cell dynamics following an onset of MI (acute and subacute phases) as a biomarker of cardiac vessel injury and myocardial injuries. Third, the average age of our control group was younger than the study groups, which may alter the results due to the age-dependent senescence of hematopoietic cells or clonal hematopoiesis. Finally, as the investigation of the molecular regulatory mechanisms responsible for the dynamic changes in peripheral mononuclear cell levels in AMI diabetic patients was not under the scope of this study, the signaling pathways underlying the observed reduction in viable cells, along with CD34+ and T cell levels in AMI patients remain unknown.

\section{Conclusion}

Our data demonstrates a significant decrease in peripheral EPCs and CD8+ T cells, three days after an AMI. Furthermore, it seems that circulating EPCs associate with the number of coronary lesions. Additionally, CD8+ T cells in the periphery (and M1-macrophages in a lower extent) may serve as high accuracy biomarkers of AMI, in combination with classical laboratory findings, with the potential to improve the dismal prognosis of AMI patients. Moreover, larger sample sized studies are warranted to confirm the ability of EPCs and CD8+ cells as potential biomarkers of cardiac vessels or myocardial injuries, respectively, for the diagnosis and prognosis of AMI.

\section{References}

1. Dutta P.; Courties G.; Wei Y.; Leuschner F.; Gorbatov R.; Robbins C.; Iwamoto Y.; Thompson B.; Carlson AL.; Heidt 
7. $\quad$ Ling L.; Shen Y.; Wang K.; Jiang C.; Fang C.; Ferro A.; Kang L.; Xu B. Worse clinical outcomes in acute myocardial infarction patients with type 2 diabetes mellitus: relevance to impaired endothelial progenitor cells mobilization. PLoS One 2012, 7.

8. $\quad$ Fadini G.P.; Miorin M.; Facco M.; Bonamico S.; Baesso I.; Grego F.; Menegolo M.; de Kreutzenberg SV.; Tiengo A.; Agostini C. et al. Circulating endothelial progenitor cells are reduced in peripheral vascular complications of type 2 diabetes mellitus. J Am Coll Cardiol 2005, 45, 1449-1457.

9. Yan X.; Anzai A.; Katsumata Y.; Matsuhashi T.; Ito K.; Endo J.; Yamamoto T.; Takeshima A.; Shinmura K.; Shen W. et al Temporal dynamics of cardiac immune cell accumulation following acute myocardial infarction. $J$ Mol Cell Cardiol 2013, 62, 24-35.

10. Leuschner F.; Rauch P.J.; Ueno T.; Gorbatov R.; Marinelli B.; Lee W.W.; Dutta P.; Wei Y.; Robbins C.; Iwamoto Y. et al. Rapid monocyte kinetics in acute myocardial infarction are sustained by extramedullary monocytopoiesis. $J$ Exp Med 2012, 209, 123-137.

11. Tsujioka H.; Imanishi T.; Ikejima H.; Kuroi A.; Takarada S.; Tanimoto T.; Kitabata H.; Okochi K.; Arita Y.; Ishibashi K. et al Impact of heterogeneity of human peripheral blood monocyte subsets on myocardial salvage in patients with primary acute myocardial infarction. J Am Coll Cardiol 2009, 54, 130-138.

12. Salybekov A.A.; Kawaguchi A.T.; Masuda H.; Vorateera K.; Okada C.; Asahara T. Regeneration-associated cells improve recovery from myocardial infarction through enhanced vasculogenesis, anti-inflammation, and cardiomyogenesis. PLoS One 2018, 13, e0203244.

13. Salybekov A.A.; Masuda H.; Miyazaki K.; Sheng Y.; Sato A.; Shizuno T.; Iida Y.; Okada Y.; Asahara T. Dipeptidyl dipeptidase-4 inhibitor recovered ischemia through an increase in vasculogenic endothelial progenitor cells and regeneration-associated cells in diet-induced obese mice. PLoS One 2019, 14, e0205477.

14. McInnes L.; Healy J.; Melville J.; UMAP: uniform manifold approximation and projection for dimension reduction. 2018, arXiv:1802.03426v2.

15. Grunkemeier G.L.; Jin R. Receiver operating characteristic curve analysis of clinical risk models. Ann Thorac Surg 2001, 72, 323-326.

16. Graber R.; Farine J.C.; Fumagalli I.; Tatti V.; Losa G.A.; Apoptosis and oxidative status in peripheral blood mononuclear cells of diabetic patients. Apoptosis 1999, 4, 263-270.

17. Fetterman J.L.; Holbrook M.; Westbrook D.G.; Brown J.A.; Feeley K.P.; Bretón-Romero R.; Linder E.A.; Berk B.D.; Weisbrod R.M.; Widlansky M.E. et al. Mitochondrial DNA damage and vascular function in patients with diabetes mellitus and atherosclerotic cardiovascular disease. Cardiovasc Diabetol 2016, 15, 1-7.

18. Moganti K.; Li F.; Schmuttermaier C.; Riemann S.; Klüter H.; Gratchev A.; Harmsen M.C.; Kzhyshkowska J. Hyperglycemia induces mixed M1/M2 cytokine profile in primary human monocyte-derived macrophages. Immunobiology 2017, 222, 952-959.

19. Uziel O.; Singer J.A.; Danicek V.; Sahar G.; Berkov E.; Luchansky M.; Fraser A.; Ram R.; Lahav M. Telomere dynamics in arteries and mononuclear cells of diabetic patients: effect of diabetes and of glycemic control. Exp Gerontol 2007, 42, 971-978.

20. Salybekov A.A.; Salybekova A.K.; Pola R.; Asahara T. Sonic hedgehog signaling pathway in endothelial progenitor cell biology for vascular medicine. Int J Mol Sci 2018, 19.

21. Shintani S.; Murohara T.; Ikeda H.; Ueno T.; Honma T.; Katoh A.; Sasaki K-i.; Shimada T.; Oike Y.; Imaizumi T. Mobilization of endothelial progenitor cells in patients with acute myocardial infarction. Circulation 2001, 103.

22. Barrett T.J.; Murphy A.J.; Goldberg I.J.; Fisher E.A. Diabetes-mediated myelopoiesis and the relationship to cardiovascular risk. Ann N Y Acad Sci 2017, 1402, 31-42.

23. Fadini G.P.; Ciciliot S.; Albiero M. Concise review: perspectives and clinical implications of bone marrow and circulating stem cell defects in diabetes. Stem Cells 2017, 35, 106-116.

24. Kojima H.; Kim J.; Chan L. Emerging roles of hematopoietic cells in the pathobiology of diabetic complications. Trends Endocrinol Metab 2014, 25, 178-187. 
25. Ma F.; Feng J.; Zhang C.; Li Y.; Qi G.; Li H.; Wu Y.; Fu Y.; Zhao Y.; Chen H. et al: The requirement of CD8+ T cells to initiate and augment acute cardiac inflammatory response to high blood pressure. J Immunol 2014, 192, 3365 3373.

26. Forteza M.J.; Trapero I.; Hervas A.; de Dios E.; Ruiz-Sauri A.; Minana G.; Bonanad C.; Gomez C.; Oltra R.; Rios-

\section{Acknowledgments}

We thank Dr. Sheng Yin for his technical support and Tokai University School of Medicine and Research Support Center team for excellent technical assistance.

\section{Authors Information}

Department of Advanced Medicine Science, Tokai University School of Medicine, Isehara, Kanagawa, Japan.

Amankeldi A. Salybekov, Kosit Vorateera and Takayuki Asahara

Department of Cardiology, Tokai University School of Medicine, Isehara, Kanagawa, Japan.

467 Katsuaki Sakai, Makoto Natsumeda and Yuji Ikari

468 Clinical Research Center, Shonan Kamakura General Hospital, Kamakura, Kanagawa, Japan.

469 Amankeldi A. Salybekov and Shuzo Kobayashi 


\section{Author Contributions}

474 Conceptualization, A.A.S, T.A., and Y.I.; methodology, A.A.S., K.S., and M.N.; software, A.A.S.; validation, 475 A.A.S., and K.S.; formal analysis, A.A.S.; investigation, A.A.S, K.V., K.S., and M.N.; resources, A.A.S., and 476 S.K.; data curation, A.A.S, K.V., K.S., and M.N.; writing—original draft preparation, A.A.S.; writing—review 477 and editing, T.A., and Y.I.; visualization, A.A.S.; supervision, T.A, and Y.I.; project administration, A.A.S.; 478 funding acquisition, T.A, S.K, and Y.I. All authors have read and agreed to the published version of the 479 manuscript.

\section{Ethics declarations}

481 This study was conducted in accordance with the Declaration of Helsinki, and approved by the Tokai 482 Clinical Investigation Ethics Committee (IRB n $\left.{ }^{\circ} 16 \mathrm{R}-026\right)$. Written informed consent was obtained from all 483 subjects prior to inclusion in the study

\section{Conflicts of Interest}

485 The authors declare no conflict of interest.

\section{Funding}

487 This research was funded by AMED (to Dr. T.A grant \#18bk0104069s0102) and the first president fund 488 BOLASHAQ to Dr. A.A.S. 


\section{Figure 1}
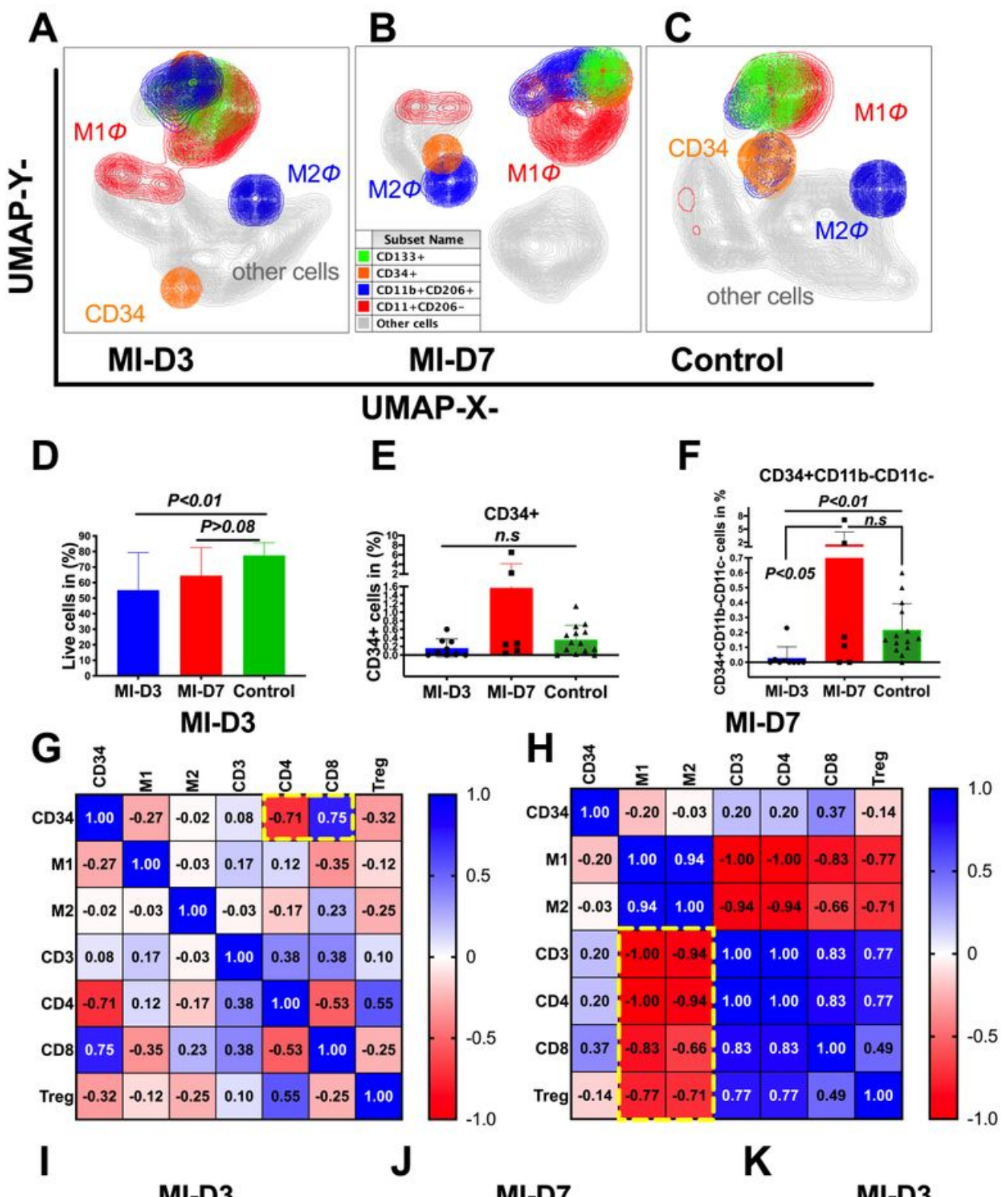

MI-D3

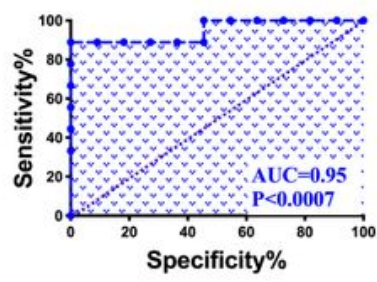

MI-D7

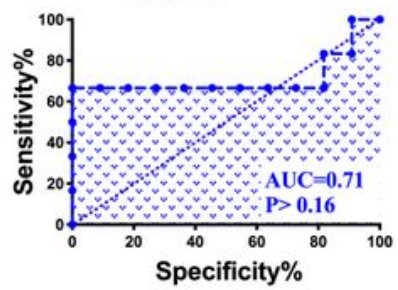

MI-D3

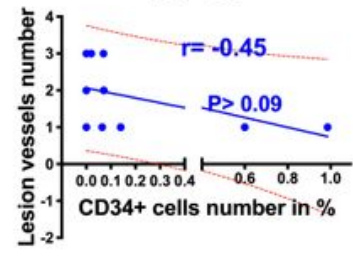

Figure 1

EPCs as a predictor of heart-vessel lesions. Unsupervised UMAP clustering method of immune cell populations were applied to distinguish myeloid and stem progenitor cells subsets at days three (A), and seven (B) after AMI onset, and in healthy controls (C). Peripheral blood live mononuclear cell frequencies 
were determined and are represented for the three groups (D). CD34+ cell frequencies are also represented (E), as are the ones for CD34+CD11b-CD11C- cells (F) for the three groups. Multivariable immune cells' correlation matrix analysis followed by the Spearman's rank test was performed and is represented 167 for patient samples at days three $(\mathrm{G})$ and seven $(\mathrm{H})$ post-AMI onset. ROC curves were designed using CD34+ cells/mL counts from AMI-D3 (I) and AMI-D7 patients (J) versus healthy controls. A correlation between CD34+ cell frequencies and cardiac vessel lesions in AMI-D3 patients was investigated and is represented $(K)$. Data are represented as group mean \pm SEM. Statistical analysis was performed using the Kruskal-Wallis test followed by a Benjamini, Krieger and Yekutieli post hoc analysis. Abbreviations: AMI-D3, 3 days post-AMI onset; AMI-D7, 7 days post-AMI onset; AMI, acute myocardial infarction; AUC, area under the receiver operating characteristics' curve

\section{Figure 2}

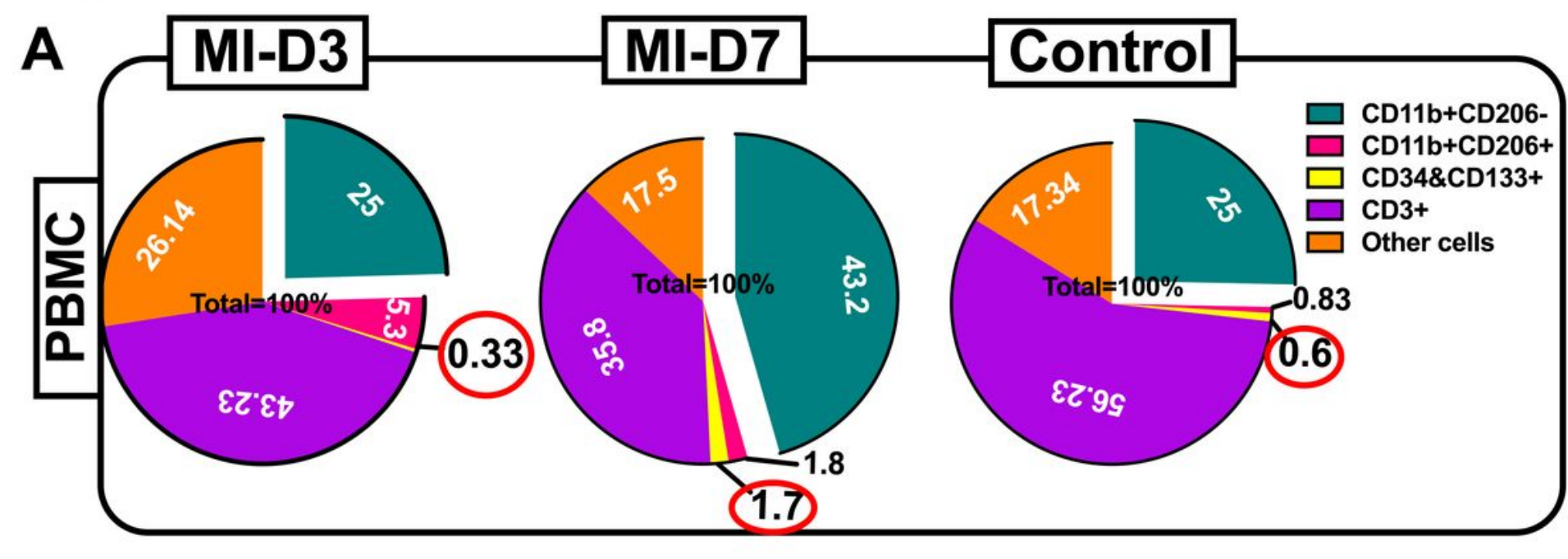

B

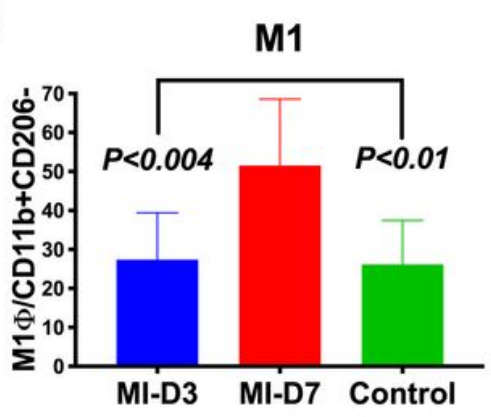

E

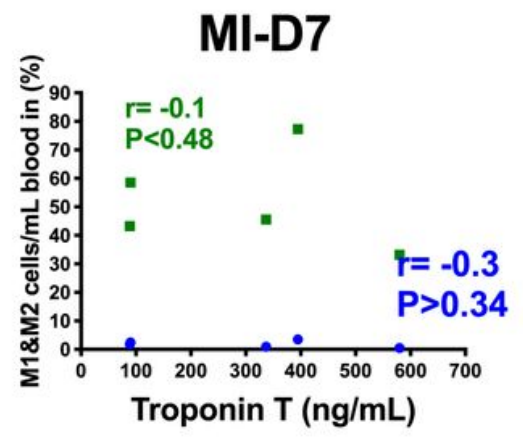

C

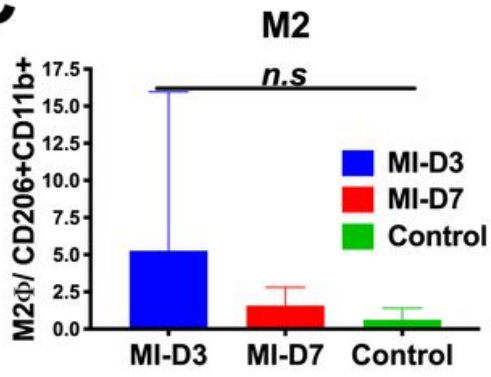

F

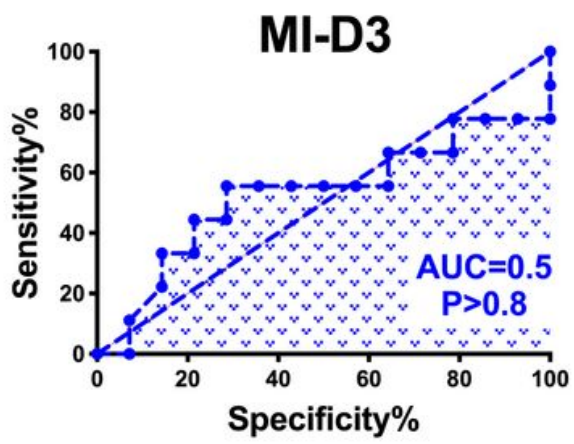

MI-D3

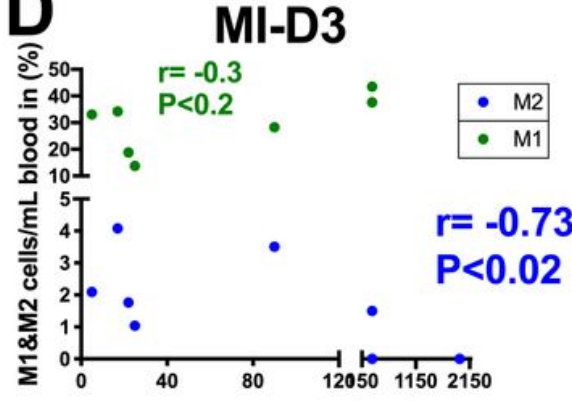

Troponin T (ng/mL)

G

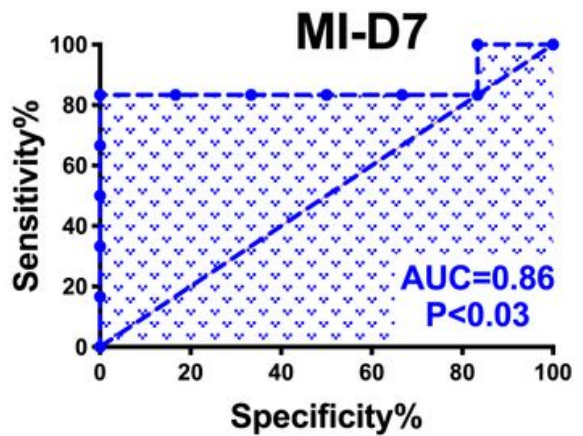




\section{Figure 2}

Macrophage subsets temporal dynamics in peripheral blood following AMI. Pie charts show myeloid cell subsets relative distribution over time in AMI patients, compared with healthy controls (A). Frequencies of (M2-) alternatively activated (B) or (M1-) classical inflammatory (C) macrophages were determined and are represented. Macrophage subsets (M1 in green and M2 in blue) were correlated with the cardiac necrosis marker Troponin T levels at days three (D) and seven (E) after AMI onset. ROC curve analysis comparing M1-macrophages from healthy individuals with AMI patients at days $3(\mathrm{~F})$ and 7 (G) post-AMI onset were performed and are represented. Data are shown as group means \pm SEM. Statistical analysis was performed using the Kruskal-Wallis test with Benjamini, Krieger and Yekutieli post hoc analysis. The Spearman rank correlation test was also used. 


\section{A B $\quad$ C}
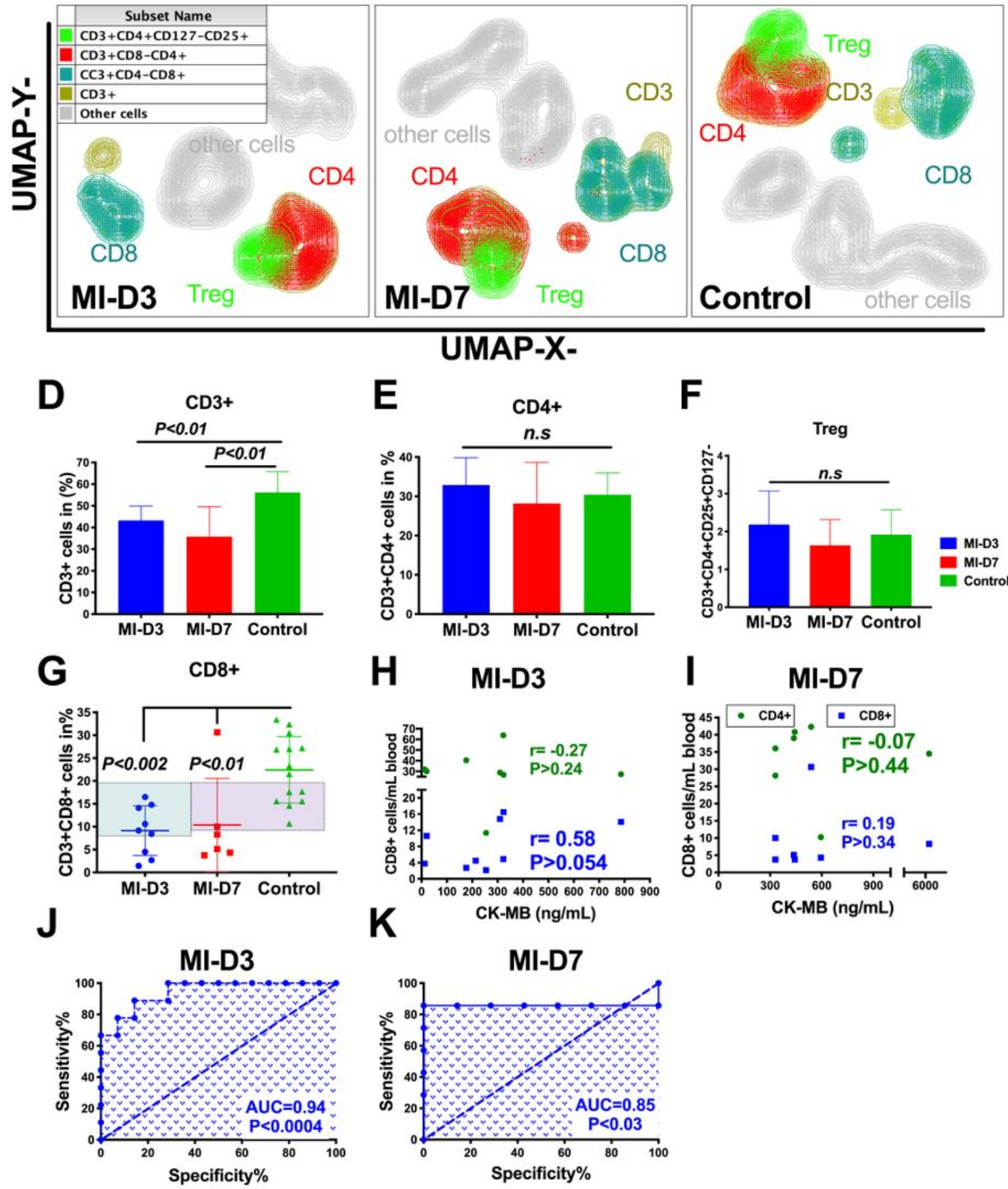

K

CK-MB (ng/mL)
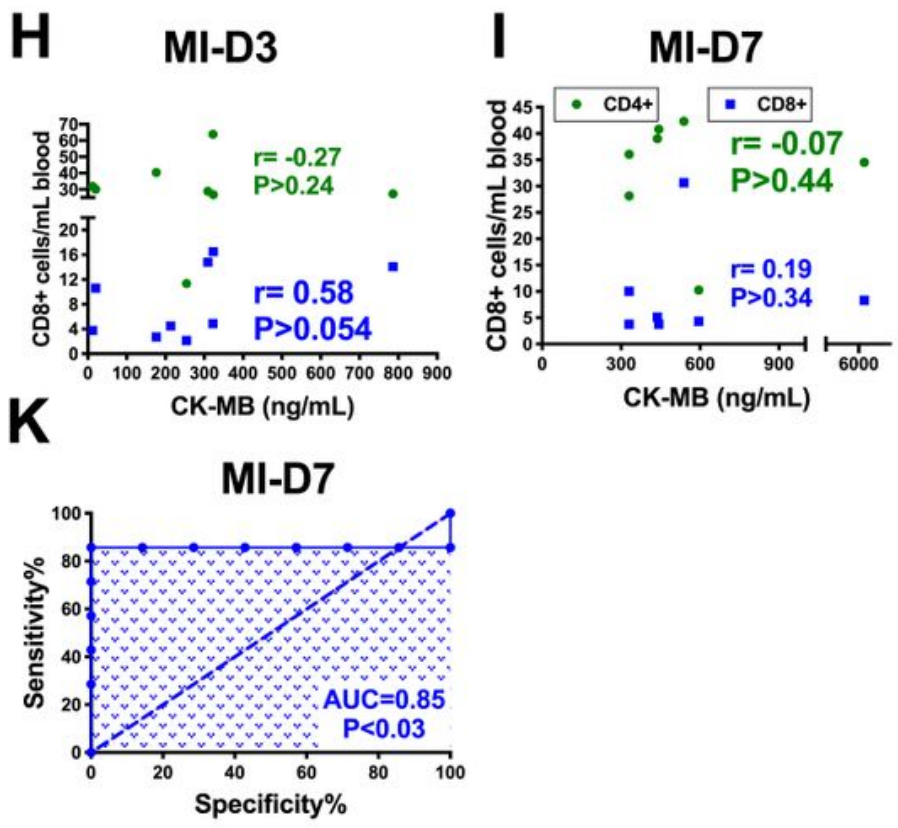

Figure 3

CD8 cells as early cardiac damage markers in AMI. The unsupervised UMAP clustering method of cell populations were applied to evaluate $T$ cell subsets at days three (A), and seven (B) post-AMI, and in healthy controls (C). Total T cell frequencies were determined in the three groups and are represented (D). Helper T cells, (E) regulatory T cells (F), and cytotoxic T cells' (G) frequencies are also represented, per group. Correlation between $\mathrm{CD} 4+$ or $\mathrm{CD} 8+\mathrm{T}$ and the cardiac necrosis marker CK-MB were investigated 
and are represented for the time-points of $3(\mathrm{H})$ and 7 (I) days after AMI onset. h). ROC curve analysis for CD8+ T cells is also represented for $3(\mathrm{~J})$ and $7(\mathrm{~K})$ days post-AMI onset. Data are shown as group means \pm SEM. Statistical analysis was performed using the Kruskal-Wallis test with Benjamini, Krieger and Yekutieli post hoc analysis. The spearman rank correlation test was also used.

\section{Figure 4}

A

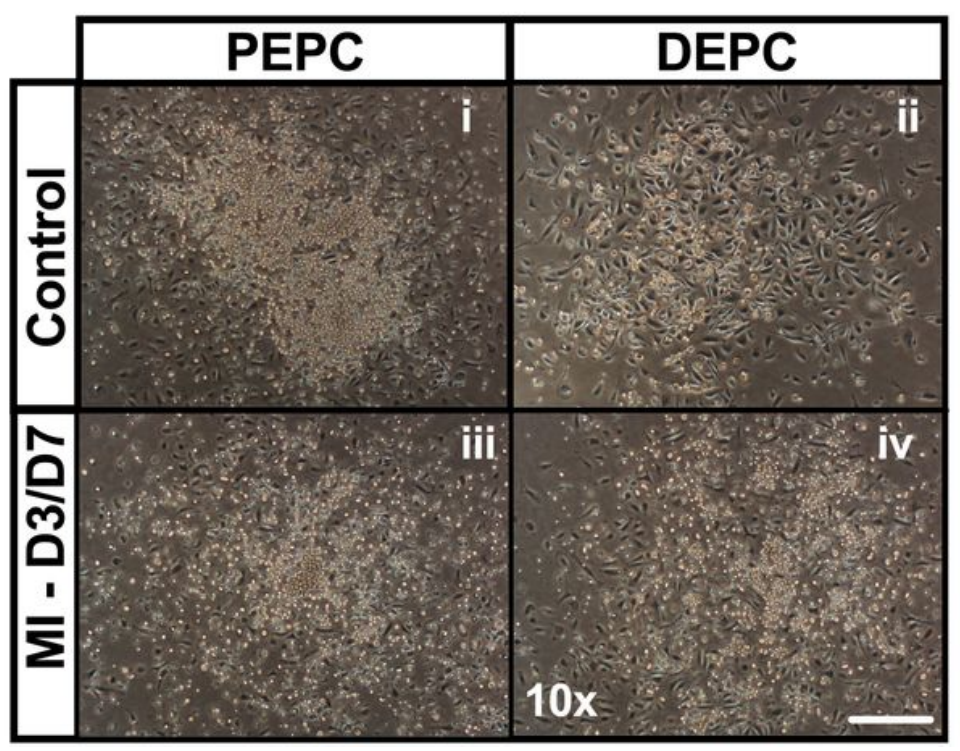

B

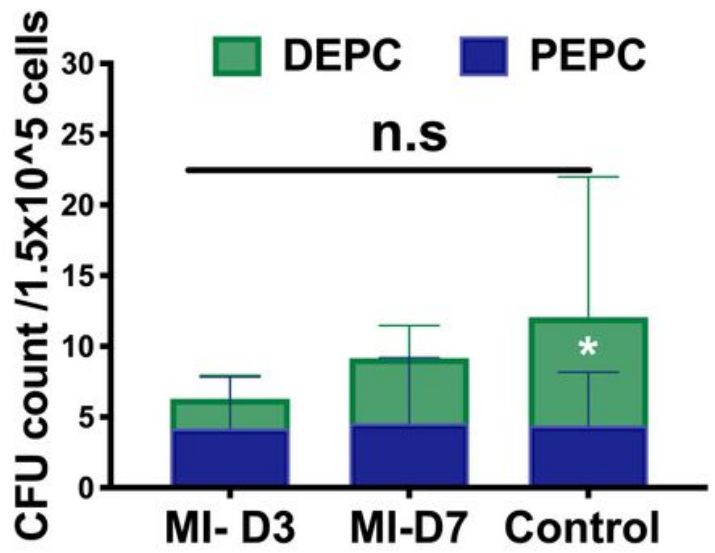

Figure 4

EPCs of an AMI patient show reduced colony formation ability. Colony-forming units of primitive EPC colonies (PEPC) and definitive EPC colonies (DEPC) were evaluated. PEPC and DEPC illustrative images are represented (A). Quantification and comparison of PEPC and DEPC among MI-D3, MI-D7, and Control groups is also represented (B). Data are shown as group means \pm SEM. Statistical analysis was performed using the Kruskal-Wallis test with Benjamini, Krieger and Yekutieli post hoc analysis.

\section{Supplementary Files}

This is a list of supplementary files associated with this preprint. Click to download.

- Suppplementarymat.docx 